\title{
OPTIMIZATION OF CNC TURNING PARAMETERS FOR AL-6061 USING RESPONSE SURFACE METHODOLOGY
}

\author{
NAVEEN KUMAR NAYAK ${ }^{1} \&$ HARSIMRAN SINGH SODHI ${ }^{2}$ \\ ${ }^{1}$ Research Scholar, Department of Mechanical Engineering, Chandigarh University, Gharuan, Mohali, Punjab, India \\ ${ }^{2}$ Assistant Professor, Department of Mechanical Engineering, Chandigarh University, Gharuan, Mohali, Punjab, India
}

\begin{abstract}
As per the present manufacturing scenario, focus of all manufacturing organizations, is to produce good quality product with a minimum cost. CNC turning process is the most common machining process that is used in now days.

Present work has been done, to optimize the machining parameters, for material AL 6061 like, depth of cut, feed rate and cutting speed. MINITAB software is used for formulating the matrix and, for the analysis of regression and response surface methodology.
\end{abstract}

KEYWORDS: CNC Turning Machine, Aluminium 6061, Minitab, Material Removal Rate \& Surface Roughness

Received: Jun 20, 2017; Accepted: Jul 10, 2017; Published: Jul 14, 2017; Paper Id: IJMPERDAUG201714

\section{INTRODUCTION}

Machining is the most widely used process for removing the unwanted material for a desired product. In the manufacturing industry, a pure alloy is required for a good quality product and at minimum manufacturing cost. Aluminium alloy 6061, is most widely used alloy, for the machining and manufacturing of a desired dimensions and shapes, aluminium 6061 alloy is preferred because, it is a lightweight metal and having good machining properties, good toughness, mechanical and thermal properties. In this research work, aluminium 6061 is considered for MRR, by varying the various process parameters like depth of cut, cutting speed and feed rate. Lot of researchers are working for finding, an optimized process, for material removal rate [1]. Jasvir Singh et al. experimentally observed, during dry turning process, the development in various machining parameters like feed rate, depth of cut and cutting speed. They performed the machining on aluminium 6061, with CNMG 120408 ENTM (H20TI) tool. To get regression equation and for optimization of the parameters RSM is used. In the experiment, they found that cutting speed and depth of the cut, gets minimum effect whereas, the feed rate gets the maximum effect on surface roughness [2]. VikasDhiman et al. presented that, the development and growth of any material depends on its machining. In this study, the various process parameters like cutting speed, depth of cut and feed rate were determined for machining on SS202 material, which shows the impact of material removal rate and surface roughness, by using L9 orthogonal array. Taguchi methodology and ANOVA is applied to analyse the effect of various parameters, on the surface roughness and material removal rate, and the experiment was conducted by Standard Orthogonal Array. In the experiment, they found the result of ANOVA (analysis of variance), using MINITAB software that reported that, mathematical model can approximately describe, the factors within the limits [3]. Joshi et al. 2012 performed the experiment CNC Vertical End Milling on aluminium, for Material removal rate. They said that, material removal process is important for producing different component 
and difficult shapes. In the end of milling operation, the end mill cutter is used, for the removal of material form work piece. End milling operation is performed, on the basis of three parameters the feed rate, the cutting speed and the depth of cut, along with it, also search the material removal rate by performing a standard orthogonal array, using Taguchi method. In the experiment, they found the result of ANOVA (analysis of variance) reported that, mathematical model can approximately describe the factors, within the limits [4]. Yadav et al. 2012 reported, surface roughness of Medium Carbon Steel AISI 1045, on turning in dry condition. In this experiment, investigated increment of machining parameters like spindle speed, depth of cut and feed rate for the Ra (surface roughness). In the experiment, the result will be found by an L27 orthogonal array, S/N (Signal to noise) and by the ANOVA (analysis of variance). The result shows that, the most significant factors which affect the surface roughness, is feed rate which is followed by depth of cut were as, the cutting speed is the least affecting factor [5]. Amity Joshi et al. 2013 studied that; the material removal process will be the most affected factor, on manufacturing the product. CNC's End Milling is the most broadly utilized technique, for machining process and the material is removed, by the cutting tool to get desired shape and design. In the experiment work, on the three basic parameters, like cutting speed, feed rate, depth of cut and use of Taguchi method, is used to find the impact on the surface finish. Analysis of variance is used, with a standard orthogonal array that shows that most significant factor is feed rate of surface finish [6]. Hemantsinh Pratapsinh Rao et al., studied the impact of depth of cut, feed rate and cutting speed on MRR (material removal rate) and Ra (surface roughness), using aluminium 6061 alloy through the Taguchi methodology. The result shows that, the cutting speed has a greater impact on Ra (surface roughness) and material removal rate (MRR), which is followed by the depth of cut [7]. Abhishek Kumbhar et al. reported the optimization of machining parameter. They also investigated, the feed rate cutting speed and depth of cut, for material removal rate and surface roughness, on SS304 using end milling machine and they also investigate, the grey relational analysis with the Taguchi methodology [8]. Himanshu Sonar et al. investigated the suitable parameters for machining, for surface roughness and material removal rate. In this experiment, he studied and carried out the parameters like cutting speed, depth of cut and feed rate for aluminium material, in CNC turning machine. Three machining tool are used, for material removing and for surface finish using L9 orthogonal array, in Taguchi methodology. Aluminium alloy 6061 experiment result will be carried out, in Microsoft excel used for regression investigation. The results show that, the machining parameters are acceptable [9].

The above literature survey shows that, the various parameters like cutting speed, depth of cut and feed rate affect the result and varying different, result in different parameter's value.

\section{EXPERIMENTAL SETUP}

CNC turning center is the most used machining process, for the accuracy and for high quality products. CNC turning center, is a machining process that is used for the removal of undesirable material from the work-piece, to give the material a desired shape and size. In this experimental setup, Aluminium 6061 is used for machining. In the experiment, minimum surface roughness and the higher material removal rate is required, for the estimation of low cost product. Optimized process for high quality product which are cost effective, is calculated by using response surface methodology, by varying the various parameters discussed below in table1: -

Table 1: Process Parameters and Limits

\begin{tabular}{|l|c|c|}
\hline Machining Parameters & Lower Limit & Upper Limit \\
\hline Feed rate $(\mathrm{mm} / \mathrm{min})$ & 78 & 102 \\
\hline Cutting speed $(\mathrm{rpm})$ & 1200 & 1800 \\
\hline
\end{tabular}




\begin{tabular}{|l|c|c|}
\hline Depth of cut $(\mathrm{mm})$ & 0.4 & 0.6 \\
\hline
\end{tabular}

\section{Below is an Adopted Procedure Followed for Experiment}

- Cut the aluminium alloy 6061 by power saw, to the desired dimensions (100 mm length).

- Measured the weight of each sample, by using highly accurate digital weighing machine before machining.

- Prepared the CNC turning code and then performed turning operation, on each sample by varying the cutting speed, the feed rate and the depth of cut.

- After machining, measured the weight of each sample using digital weighing machine.

- $\quad$ Calculated the MRR as: -

- $\quad \mathrm{MRR}=($ initial weight - final weight $) \div($ density $\times$ time $)$

- Measured the surface roughness with the help of a portable stylus type Profilometer "Talysurf".

- $\quad$ Using MINITAB software, RSM statics was analysed.

- A matrix was formulated, for the experimentation is shown in the below table 2

Table 2: Matrix Formulation for Experiment

\begin{tabular}{|c|c|c|c|c|}
\hline $\begin{array}{c}\text { Std } \\
\text { Order }\end{array}$ & $\begin{array}{c}\text { Run } \\
\text { Order }\end{array}$ & $\begin{array}{c}\text { Cutting } \\
\text { Speed(rpm) }\end{array}$ & $\begin{array}{c}\text { Feed rate } \\
(\mathbf{m m} / \mathbf{m i n})\end{array}$ & $\begin{array}{c}\text { Depth of cut } \\
(\mathbf{m m})\end{array}$ \\
\hline 4 & 1 & 1800.00 & 102.000 & 0.400000 \\
\hline 14 & 2 & 1500.00 & 90.000 & 0.668179 \\
\hline 16 & 3 & 1500.00 & 90.000 & 0.500000 \\
\hline 13 & 4 & 1500.00 & 90.000 & 0.331821 \\
\hline 3 & 5 & 1200.00 & 102.000 & 0.400000 \\
\hline 19 & 6 & 1500.00 & 90.000 & 0.500000 \\
\hline 15 & 7 & 1500.00 & 90.000 & 0.500000 \\
\hline 7 & 8 & 1200.00 & 102.000 & 0.600000 \\
\hline 10 & 9 & 2004.54 & 90.000 & 0.500000 \\
\hline 11 & 10 & 1500.00 & 69.818 & 0.500000 \\
\hline 17 & 11 & 1500.00 & 90.000 & 0.500000 \\
\hline 12 & 12 & 1500.00 & 110.182 & 0.500000 \\
\hline 6 & 13 & 1800.00 & 78.000 & 0.600000 \\
\hline 8 & 14 & 1800.00 & 102.000 & 0.600000 \\
\hline 2 & 15 & 1800.00 & 78.000 & 0.400000 \\
\hline 20 & 16 & 1500.00 & 90.000 & 0.500000 \\
\hline 1 & 17 & 1200.00 & 78.000 & 0.400000 \\
\hline 18 & 18 & 1500.00 & 90.000 & 0.500000 \\
\hline 9 & 19 & 995.46 & 90.000 & 0.500000 \\
\hline 5 & 20 & 1200.00 & 78.000 & 0.600000 \\
\hline & & & & \\
\hline
\end{tabular}

\section{RESULT AND ANALYSIS}

Following results have been found after the experiment, that shows in the below table 3

Table 3: Experiment Result Data 


\begin{tabular}{|c|c|c|c|c|c|c|c|c|}
\hline $\begin{array}{c}\text { SI. } \\
\text { No. }\end{array}$ & $\begin{array}{c}\text { Cutting } \\
\text { Speed } \\
(\mathbf{r p m})\end{array}$ & $\begin{array}{c}\text { Feed } \\
\text { Rate } \\
(\mathbf{m m} / \mathbf{m i n})\end{array}$ & $\begin{array}{c}\text { Depth of } \\
\mathbf{C u t} \\
(\mathbf{m m})\end{array}$ & $\begin{array}{c}\text { Initial } \\
\text { Weight } \\
(\mathbf{g})\end{array}$ & $\begin{array}{c}\text { Final } \\
\text { Weight } \\
(\mathbf{g})\end{array}$ & $\begin{array}{c}\text { Time } \\
(\mathbf{s e c} .)\end{array}$ & $\begin{array}{c}\text { MRR } \\
(\mathbf{m m} / \mathbf{s e c})\end{array}$ & Ra $(\boldsymbol{\mu m})$ \\
\hline 1 & 1800 & 102 & 0.4 & 137.87 & 133.36 & 38 & 43.9 & 1.045 \\
\hline 2 & 1500 & 90 & 0.668179 & 136.06 & 129.97 & 40.78 & 55.3 & 0.4155 \\
\hline 3 & 1500 & 90 & 0.5 & 136.33 & 131.55 & 40.23 & 44 & 0.4355 \\
\hline 4 & 1500 & 90 & 0.331821 & 138.74 & 134.96 & 41.48 & 33.75 & 0.5615 \\
\hline 5 & 1200 & 102 & 0.4 & 137.16 & 133.09 & 37.16 & 40.56 & 0.498 \\
\hline 6 & 1500 & 90 & 0.5 & 137.27 & 132.44 & 39.99 & 44.73 & 0.558 \\
\hline 7 & 1500 & 90 & 0.5 & 137.32 & 132.34 & 40.43 & 45.62 & 0.369 \\
\hline 8 & 1200 & 102 & 0.6 & 136.98 & 131.3 & 35.15 & 59.84 & 0.471 \\
\hline 9 & 2004.54 & 90 & 0.5 & 135.81 & 130.95 & 40.18 & 44.79 & 0.3975 \\
\hline 10 & 1500 & 69.818 & 0.5 & 135.02 & 130 & 51.51 & 36.09 & 0.3785 \\
\hline 11 & 1500 & 90 & 0.5 & 135.97 & 131.91 & 40.17 & 37.43 & 0.4055 \\
\hline 12 & 1500 & 110.182 & 0.5 & 137.53 & 132.28 & 33.19 & 58.58 & 0.509 \\
\hline 13 & 1800 & 78 & 0.6 & 137.77 & 131.88 & 46.8 & 46.61 & 0.373 \\
\hline 14 & 1800 & 102 & 0.6 & 137.97 & 131.88 & 35.88 & 62.86 & 0.432 \\
\hline 15 & 1800 & 78 & 0.4 & 136.89 & 132.5 & 46.48 & 34.98 & 0.379 \\
\hline 16 & 1500 & 90 & 0.5 & 140.4 & 135.01 & 39.97 & 49.94 & 0.3995 \\
\hline 17 & 1200 & 78 & 0.4 & 136.99 & 132.43 & 45.81 & 36.86 & 0.3665 \\
\hline 18 & 1500 & 90 & 0.5 & 136.88 & 131.45 & 40.43 & 49.74 & 0.421 \\
\hline 19 & 995.46 & 90 & 0.5 & 136.66 & 131.2 & 40.68 & 49.71 & 0.5305 \\
\hline 20 & 1200 & 78 & 0.6 & 136.17 & 130.16 & 46.03 & 48.35 & 0.385 \\
\hline
\end{tabular}

MRR and Ra have been calculated with respect to the experimental data in table 3

\section{RSM Statics for MRR}

In the beginning checked the data by the residual plots (figure 1), this plots shows the normality of experiment. The data points as shown in the graph are distributed near and along the normal line, so we called that an normal distributed result. The second plot doesn't shows any interactions between when plotting residual versus fitted value data that shows RSM model is good with the data set. Third plot residual histogram showing data distribution and last graph shows the experiment order of the data points which concerned order significance and not.

\section{Main Effect Plot for MRR}

This graph (figure 2) shows the parameters (feed rate, cutting speed and depth of cut) and effect on material removal rate, in case of cutting speed increase, when value is 1550 to $1800 \mathrm{rpm}$. At the point of 1000 to $1500 \mathrm{rpm}$, there is a sudden decrease, in the MRR. After that, increasing the cutting speed shows the increase in MRR. In case, of feed rate material removal rate is increasing continuously in all levels of feed rate. Similarly in case of depth of cut material removal rate is increasing continuously up to the $0.66 \mathrm{~mm}$, after that the MRR value is increasing slightly. 


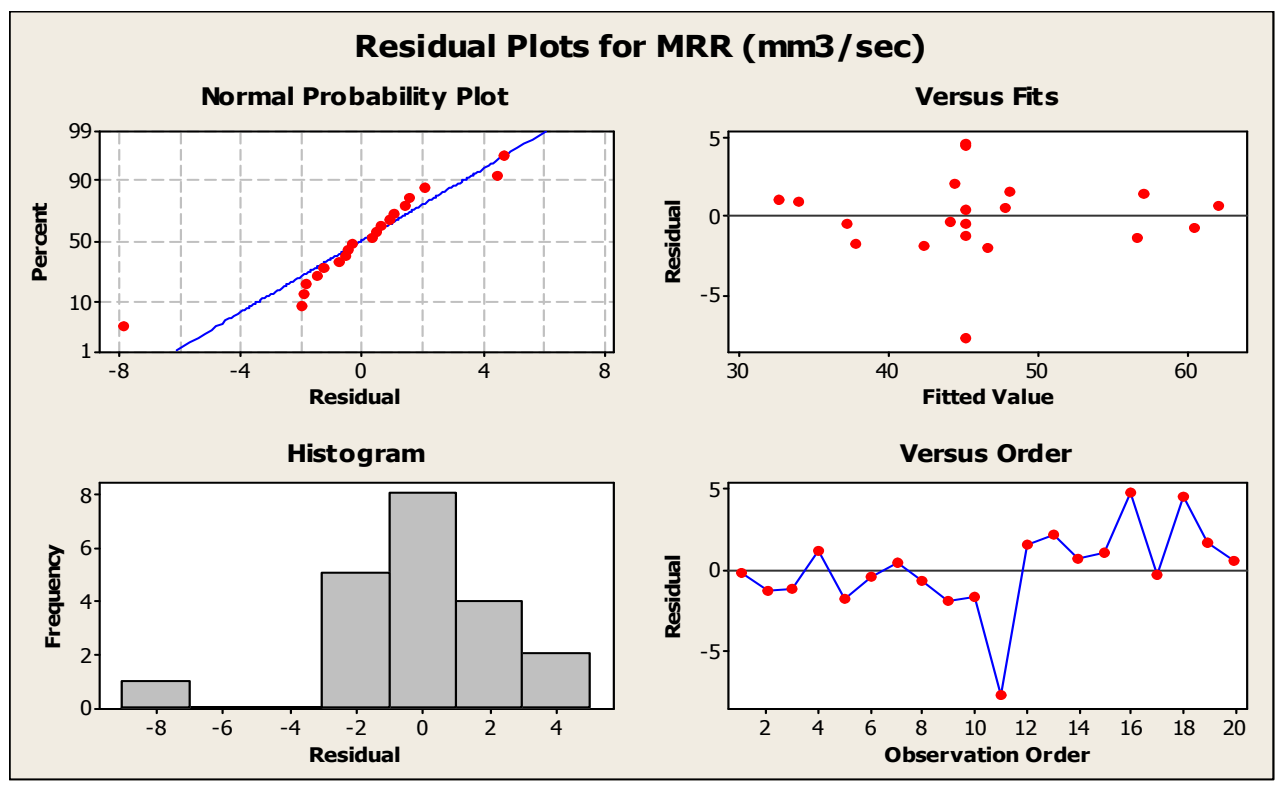

Figure 1: Probability Plot Graph for MRR

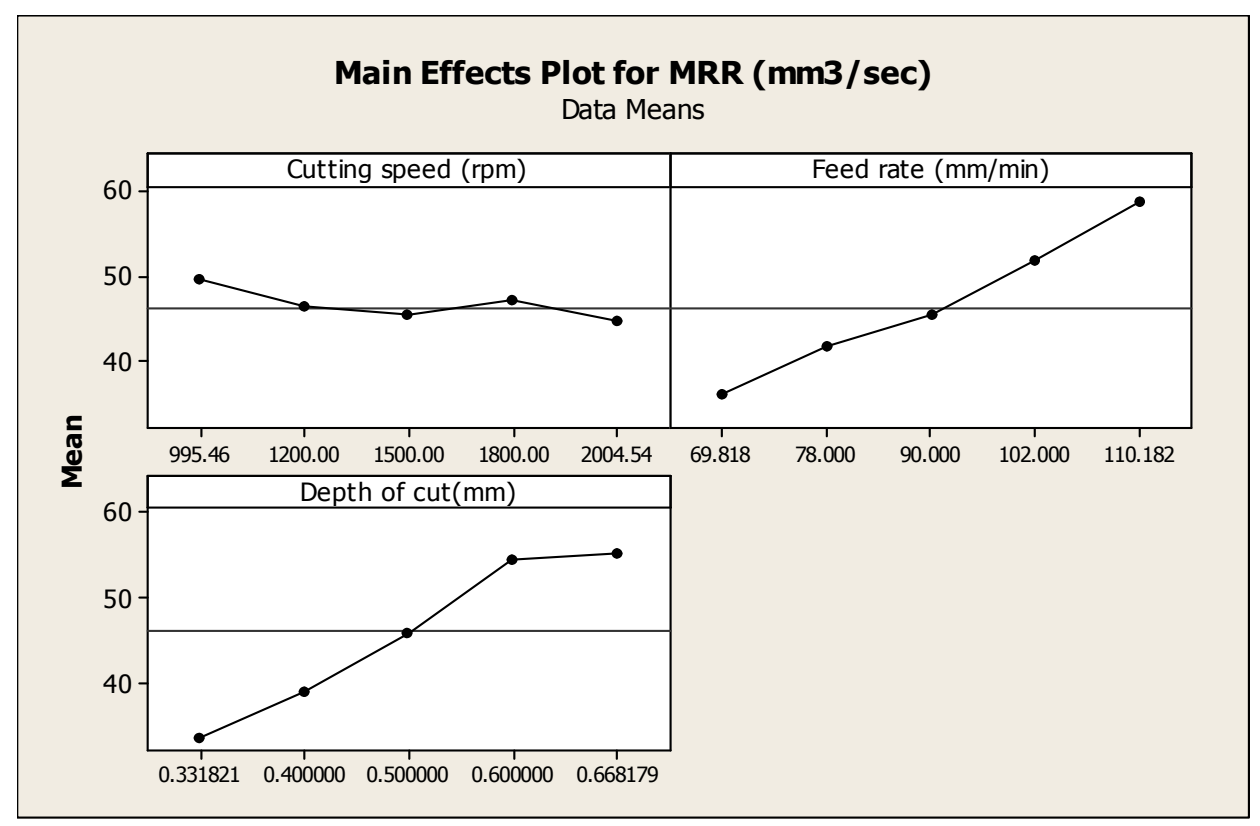

Figure 2: Main Effect Plot for MRR 
Graphical Inference of MRR

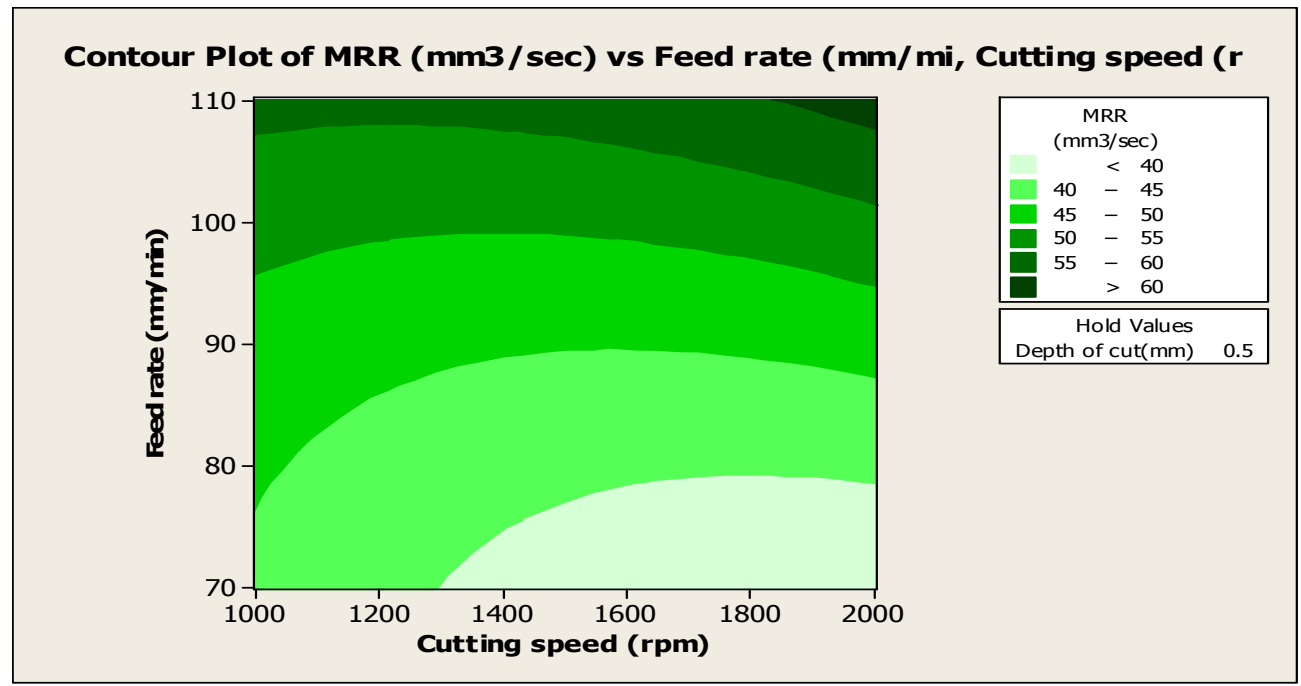

Figure 3: Contour Plot of MRR vs Feed Rate, Cutting Speed

Contour plots are shows the different colour regions on different output values. In this graph (Figure 3) dark green area shows, the cut of feed rate and depth of cut where, MRR value is maximum and above $60 \mathrm{~mm}^{3} / \mathrm{sec}$.

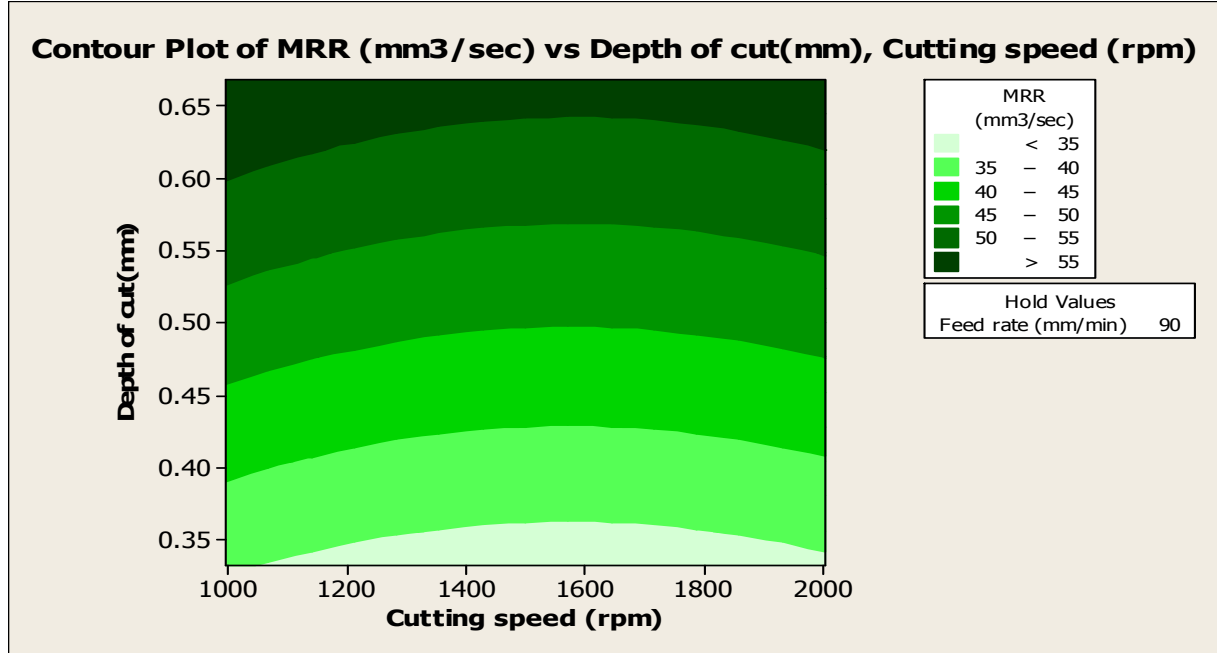

Figure 4: Contour Plot of MRR vs Depth of Cut, Cutting Speed

In the above graph (figure 4) shows the effect of cutting speed and depth of cut on Material removal rate. In the graph all the cutting speed parameters are effective when depth of cut is above the $0.60 \mathrm{~mm}$. When cutting speed $1500-1700$ $\mathrm{rpm}$ and depth of cut is $0.64 \mathrm{~mm}$ then the material removal rate is maximum above the $55 \%$. 


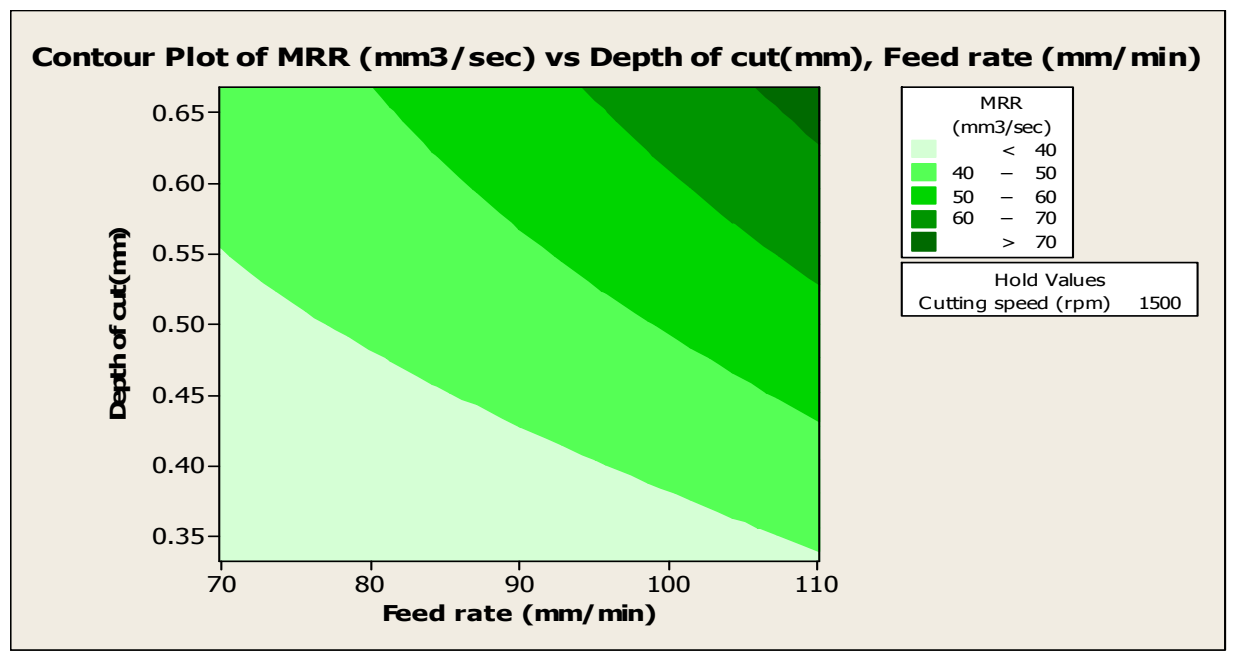

Figure 5: Contour Plot of MRR vs Depth of Cut, Feed Rate

This graph (figure 5) shows relation between depth of cut and feed rate. According to this graph material removal rate is $>70 \%$ when the depth of cut is about to $0.64-0.66 \mathrm{~mm}$ and feed rate is maximum at $108-110 \mathrm{~mm} / \mathrm{min}$ at the hold cutting speed value $1500 \mathrm{rpm}$.

\section{RSM Statics for Ra}

For minimizing surface roughness, we performed response surface methodology, using Minitab on input datas. Profilometer is used, for measuring the surface roughness. In this graph (figure 6), shows the normal probability distribution function and it shows parameters that are correct, for performing the experiment. First, normal probability plot shows, all the data point near and along, with the center line that shows the normally distribution. Second, versus fits plot does not interact with any point, shows the plotted data value is correct. Third, the histogram shows, the variation on all the parameters. Forth, observation order all data point, varying in between the line and doesn't show any interaction between any points. All the points show that, our parameters are good for the experiment.

\section{Main Effect Plot}

Figure 7, shows the parameter with efficiency of cutting speed, depth of cut and feed rate. Cutting speed shows, when speed is between 995.46-1200 rpm there is a decrease in surface roughness, when speed is 1200-1500 rpm there is a slight increase and when the cutting speed is between 1500-1800 rpm, there is maximum increase, in surface roughness. In the other words, surface roughness increases when the feed rate is between, $78-102 \mathrm{~mm} / \mathrm{min}$, in the other side, surface roughness is increasing when depth of cut is between the $0.33-0.40 \mathrm{~mm}$. 


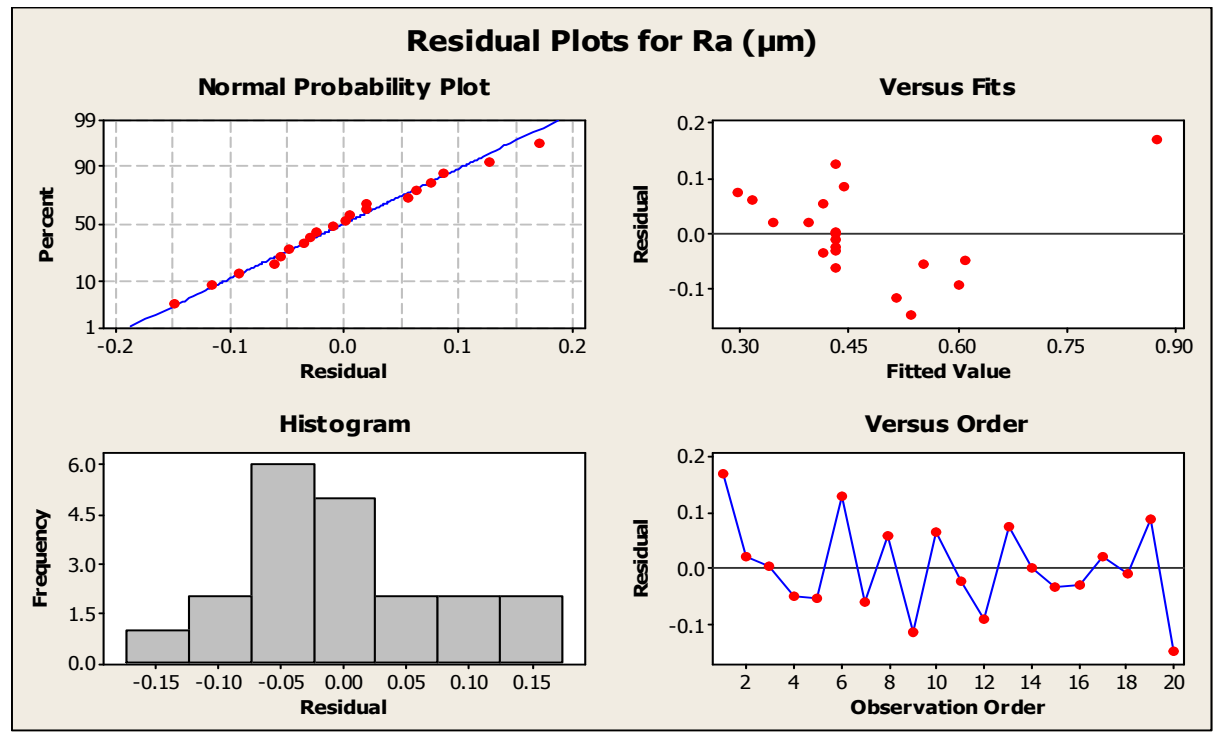

Figure 6: Residual Plot for Ra

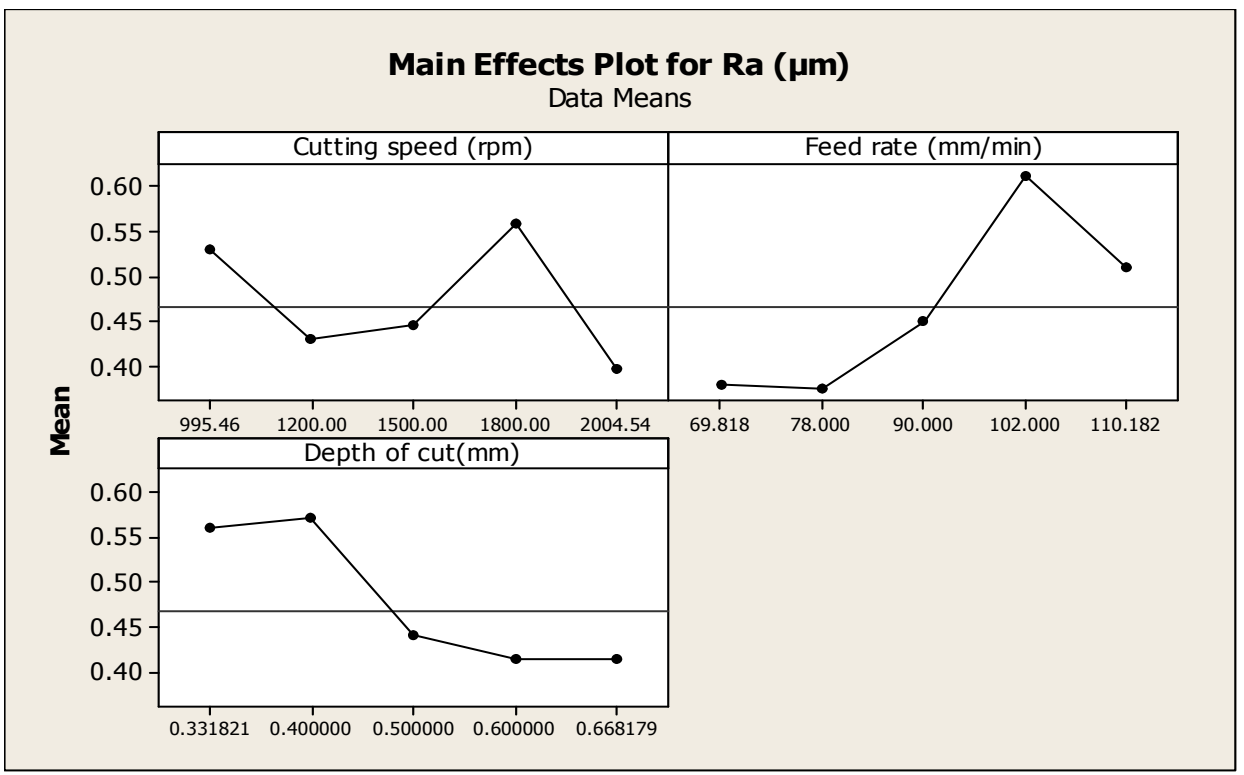

Figure 7: Main Effect Plot for Ra 


\section{Graphical Inference of Ra}

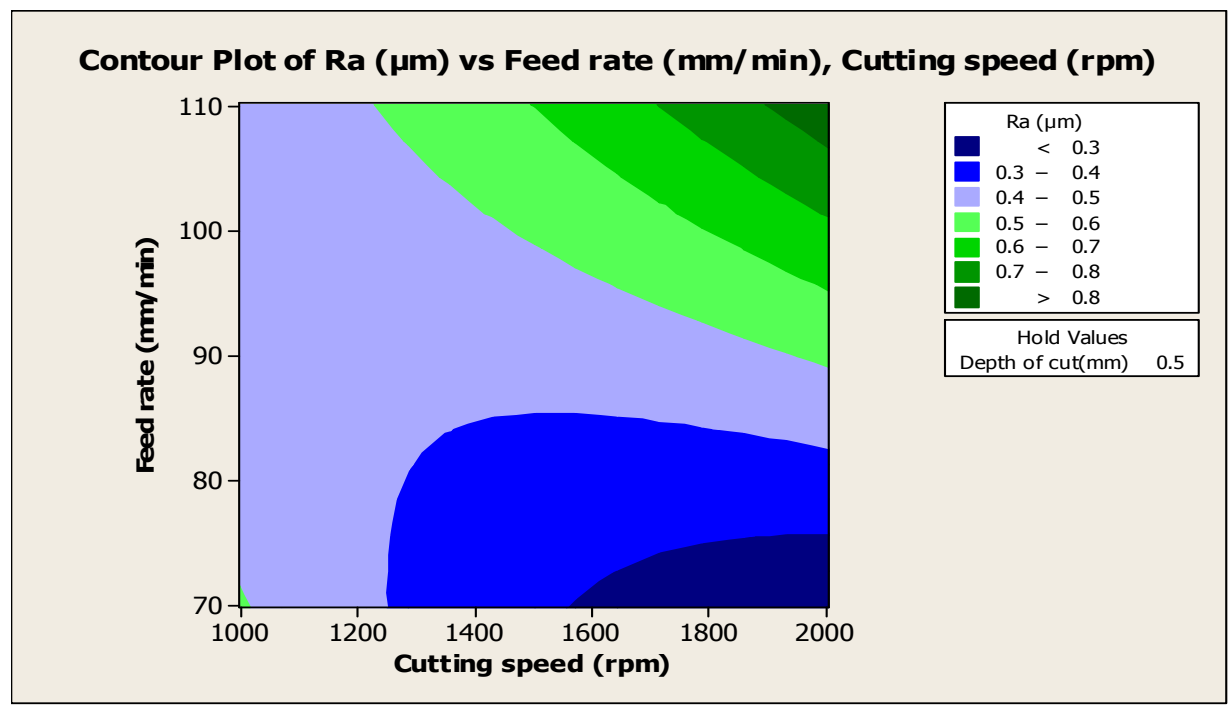

Figure 8: Contour Pot of Ra vs Feed Rate, Cutting Speed

The above graph (figure 8) shows the effect on feed rate and cutting speed, on surface roughness. The graph shows that, surface roughness is minimum $(<0.3 \mu \mathrm{m})$ when cutting speed is maximum $(1600-2000 \mathrm{rpm})$ and feed rate is minimum (70-75 mm).

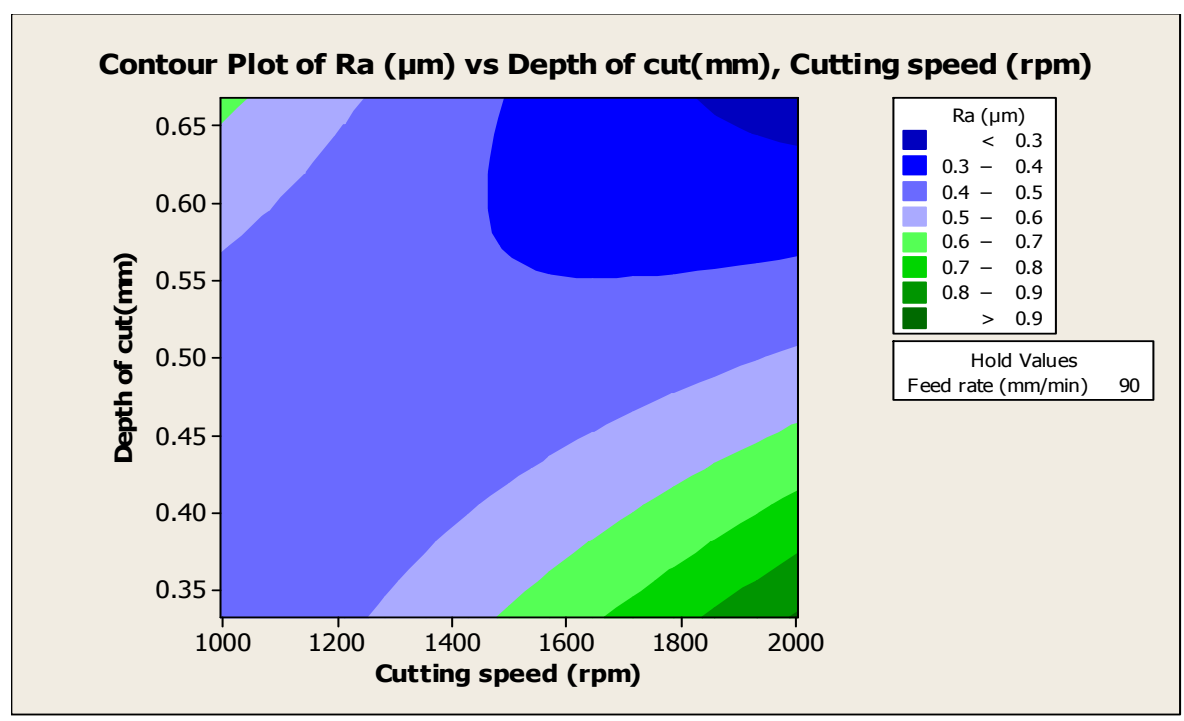

Figure 9: Contour Plot of Ra vs Depth of Cut, Cutting Speed

The above graph (figure 9), shows the response on depth of cut and cutting speed, on the surface roughness. The graph shows that, surface roughness is minimum $(<0.3 \mu \mathrm{m})$ when cutting speed is maximum $(1900-2000 \mathrm{rpm})$ and depth of cut is maximum $(0.63-0.66 \mathrm{~mm} / \mathrm{min})$. 


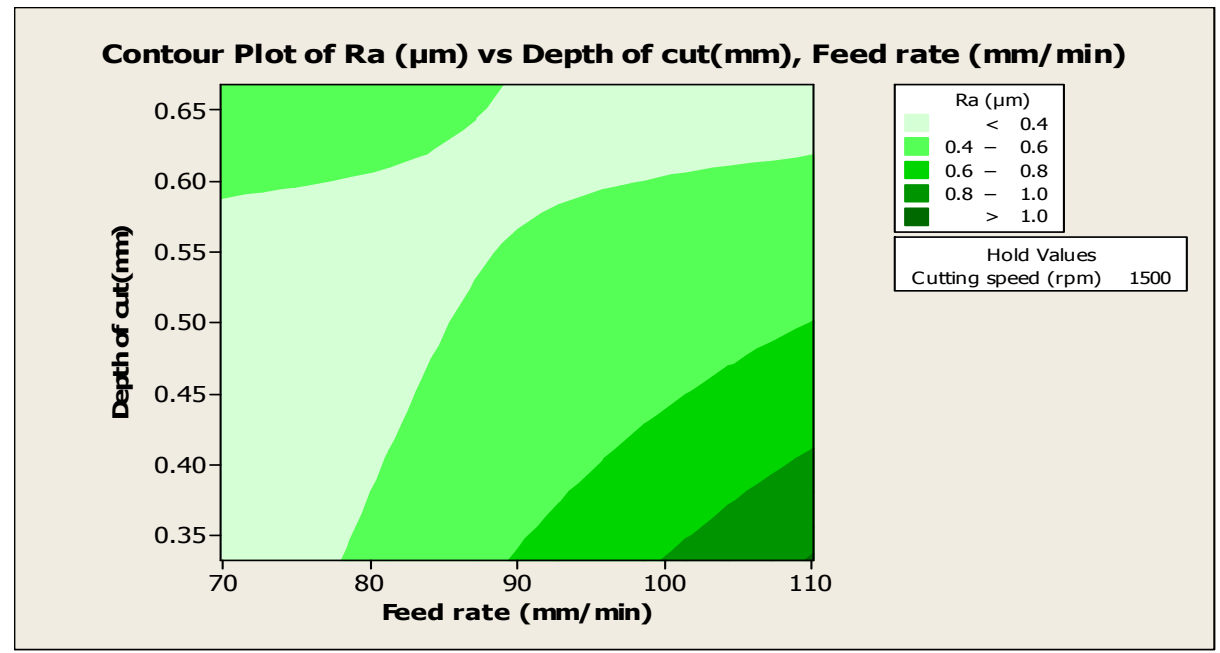

Figure 10: Contour Plot of Ra vs Depth of Cut, Feed Rate

Graph (figure 10), shows the response on depth of cut and feed rate, on surface roughness. The graph shows that, surface roughness is maximum $(>1 \mu \mathrm{m})$ when feed rate is maximum $(100-110 \mathrm{~mm} / \mathrm{min})$ and depth of cut is minimum $(0.35$ $0.43) \mathrm{mm}$.

\section{PREDICTED RESPONSE}

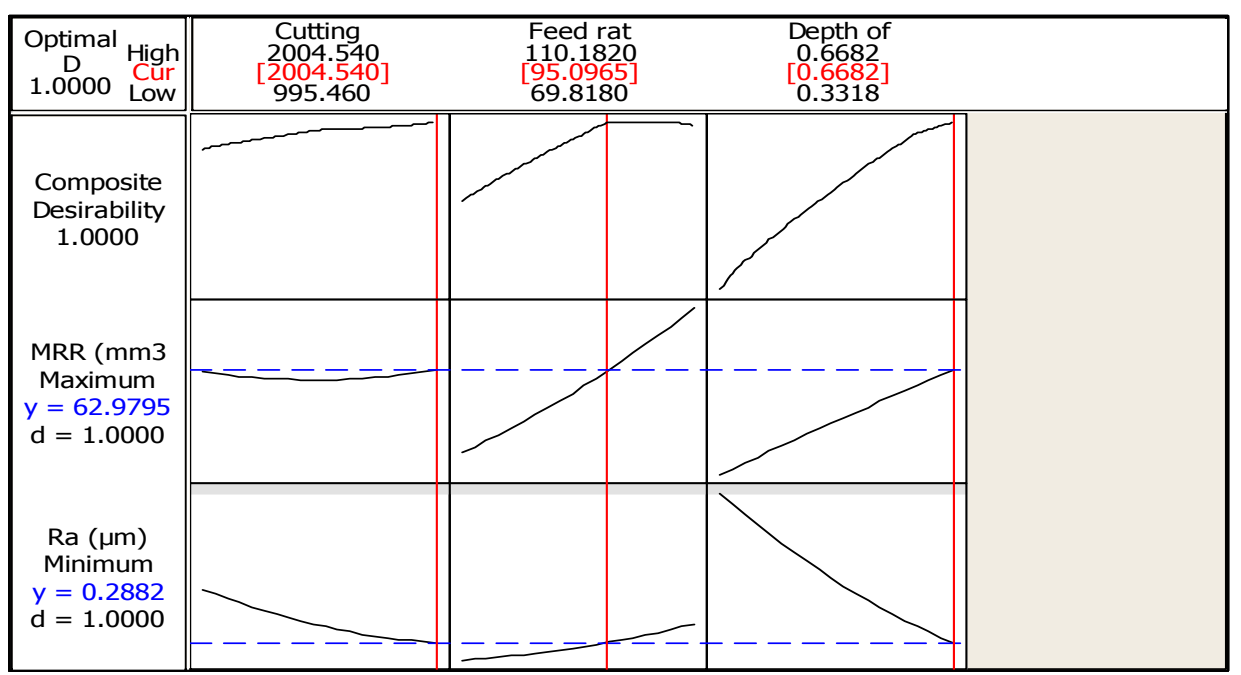

Figure 11: Predicated Responses

\section{CONCLUSIONS}

From the predicted response, it has been found that, if the machine is run at the setting of cutting speed, 2004.54 $\mathrm{rpm}$, feed rate of $95.0965 \mathrm{~mm} / \mathrm{min}$ and depth of cut to be $0.6682 \mathrm{~mm}$, then there is $100 \%$ composite desirability that we will get, on Material removal rate (MRR) of $62.9795 \mathrm{~mm}^{3} / \mathrm{sec}$ and surface roughness (Ra) of 0.2882.

\section{REFERENCES}

1. BikramJit Singh, Harsimran Singh Sodhi, "Parametric Optimization of CNC turning for AL-7020 with RSM" Internation Journal operational research, Vol. 20, No.2, 2014. 
2. Jasvir Singh, Harvinder Singh, Gagandeep Singh Dhindsa, "optimization of machining parameters during CNC turning of Aluminium 6061 with CNMG EN-TM (H20TI) insert using Response Surface Methodology (RSM)”, 2016 IJEDR volume 4 issue 1 ISSN: 2321-9939.

3. VikasDhiman, Deepak Gupta, "optimization of process parameters for material removal rate and surface roughness for SS 202 material during face milling operation” International journal for Innovative research in Science \& technology, volume 1 Issue 11 (2015) ISSN: 2349-6010.

4. A. joshi et al, "experimental investigation of machining parameters of CNC milling on material removal rate by Taguchi Method" international journal of applied engineering research,2012, volume 7 NO.11.

5. U.K. Yadav, D. narang, p.s.attri, "experimentak investigation and optimization of machining parameters for surface roughness in CNC Turning by Taguchi Method" international journal of engineering research and applications, vol. 2, Issue 4, pp.2060-2065, 2012.

6. Amitjoshi, Pradeepkothiyal, "investigating effect of machining parameter of CNC Milling on surface finish by Taguchi method" internatinaol journal on theoretical and applied research in modern engineering and emerging technology, vol. 1, Issue: 3 ISSN: 2320-6586.

7. HemantsinhpratapsinhRao, Prof. Rajat Dave, "Optimization of CNC End Milling Process Parameters for Aluminium 6061 Alloy using Carbide Tool Material by Design of Experiments" International journal for innovative research in science \& technology, volume 3, Issue 11, April 2017, ISSN : 2349-6010.

8. AbhishekKumbhar, RohitBhosale, AmitModi, ShalakaJadhav, Suresh Nipanikar, AdityaKulkarni, "Multi-objective Optimization of Machining Parameters in CNC End Milling of Stainless Steel 304" International Journal of Innovative Research in Science, Engineering and Technology Vol. 4, Issue 9, September 2015.

9. himanshu sonar, vishudhasaxena, vivekkshirsagar, shraddha p deshpande, " process parametersoptimization of CNC Turning machinen for Aluminium alloy using Taguchi method" International journal of research in engineering and technology, eISSN: 2319-1163 | pISSN: 2321-7308.

10. B.j. singh, D. khanduja, “ a case study in die casting foundry” International journal of productivity and quality management, volume 8, no.4, pp.373-397.

11. Harsimran Singh Sodhi, BikramJit Singh, Dr. Dinesh Khanduja "Behavior study of cutting parameters on Material Removal Rate for a non-ferrous material while turning on a CNC turning center." International Journal of Applied Engineering Research, ISSN 0973-4562 Vol.7 No.11 (2012) (SCOPUS Index)

12. Harsimran Singh Sodhi, DhirajPrakashDhiman, Ramesh Kumar Gupta, Raminder Singh Bhatia "Investigation of Cutting Parameters For Surface Roughness of Mild Steel In Boring Process Using Taguchi Method” International Journal of Applied Engineering Research, ISSN 0973-4562 Vol.7 No.11 (2012). (SCOPUS Index)

13. Harsimran Singh Sodhi, Harjot Singh "Parametric Analysis Of Copper For Cutting Processes Using Turning Operations Based On Taguchi Method” International Journal Of Research In Mechanical Engineering And Technology” IJRMET Vol. 3 , Issue 2, May - Oct. 2013, ISSN : 2249-5762 (Online) ISSN : 2249-5770 (Print).pp 202-204.

14. GauravVohra, Palwinder Singh, Harsimran Singh Sodhi" Analysis and Optimization of Boring Process Parameters By Using Taguchi Method" International Journal of Computer Science and Communication Engineering,IJCSCE Special issue on “Recent Advances in Engineering \& Technology” NCRAET-2013. ISSN 2319-7080.pp 232-237.

15. Gauravvohra, Harsimran Singh Sodhi, Suneev Anil Bansal "Prediction of Optimised Parameters for CNC Boring Process Using Taguchi Method for Steel” International Journal of Mechanical Science and Civil Engineering ISSN 2320-2424.pp-3136.

16. BikramJit Singh, Harsimran Singh Sodhi, (2014)"Parametric optimisation of CNC turning for Al-7020 with RSM" International Journal of operations research, Vol 20 No.2 pp 180-206. (SCOPUS Indexed Impact Factor 1.3)

17. Harjot Singh Panag, Harsimran Singh Sodhi, Harjot Singh (2014) “Optimization of Surface Roughness in EDM For AISI M2 High Speed Steel Using Response Surface Methodology” IJRMET Vol.4, Issue 2, sp l- 2 May-october 2014. PP- 139-142. 
18. GauravBansal, Harsimran Singh Sodhi, Jasmeet Singh(2014) "Optimization of Machining Parameters for MRR in Boring Operation Using RSM” IJRMET Vol.4, Issue 2, sp l- 2 May-October 2014. PP 127-131

19. Gurmeet Singh, Harjit Singh Mangat, Harsimran Singh Sodhi (2014) “Optimization of end milling process for d2 (die steel) by using Response surface methodology” Journal of Production Engineering, Vol 17. No.2 pp-73-78. (Dec 2014)

20. VarinderKhurana, Harsimransinghsodhi, Amarjeet Singh Sandhu “The Effect of Die Sinking Process Paramters on Surface roughness of D2 Steel Using Taguchi"s method" Volume 2, Issue 3, December 2014, International Journal of Innovative Research in Engineering \& Multidisciplinary Physical Sciences, ISSN: 2349-7300 ISO 9001:2008 Certified.

21. SukhmanSodhi,RupinderkaurGurm,Harsimran Singh Sodhi (2015) “Optimization of Number of Nodes for AODV, OLSR And ZRP Protocols with and Without Black Hole Attack inMANET by Using Taguchi Method" International Journal of Computer Science Trends and Technology (IJCST) - Volume 3 Issue 4, Jul-Aug 2015,ISSN: 2347-8578

22. SukhmanSodhi,RupinderkaurGurm,Harsimran Singh Sodhi (2015) “Optimization of Number of Nodes for AODV Protocol with and Without Black Hole Attack in MANET by Using Taguchi Method” International Journal of Computer Science Trends and Technology (IJCST) - Volume 3 Issue 4, Jul-Aug 2015. ISSN: 2347-8578.

23. Harsimran Singh Sodhi, Dr Bikramjit Singh, Dr Doordarshi Singh (2015) "Rate of material removal and surface roughness modelling and optimization in CNC milling for mild steel using Taguchi technique” International Journal of Scientific Research (IJSR) (ISSN No: 2277-8179),Vol 4, Issue 10,PP-104-107 the impact factor of the journal is 3.24 